\title{
Trends of Inference Considering Extended Fuzzy Logic (FLe)
}

\section{Farnaz Sabahi *}

Ferdowsi University of Mashhad, Mashhad, Iran

*Corresponding author: Farnaz Sabahi, Ferdowsi University of Mashhad, Mashhad, Iran, Tel: 34953 212 118; E-mail: farnazsabahi1@gmail.com

Rec Date: July 25 2014; Acc Date: July 26 2014; Pub Date: July 272014

Citation: Sabahi F (2014) Trends of Inference Considering Extended Fuzzy Logic (FLe). Adv Robot Autom 3: e124. doi: 10.4172/2168-9695.1000e124

Copyright:@ 2014 Sabahi F. This is an open-access article distributed under the terms of the Creative Commons Attribution License, which permits unrestricted use, distribution, and reproduction in any medium, provided the original author and source are credited.

\section{Introduction}

The introduction of fuzzy logic in 1965 has been one of the main advancements in the last several decades in different subjects and especially in control theory [1]. Fuzzy logic has given large deals to reasoning, and clearly, shows its applicability in many areas to solve different problems concerning approximate reasoning. However, in large measure what remains unrecognized is that fuzzy logic is itself a precise logic of imprecise reasoning. In fact, the definitions of membership functions and generalized constraints are precise within fuzzy logic [2]. This precision requires the necessary condition of provably validity about achieved results. This condition can be satisfied only in the problems defined in closed world assumption that can be handled by mathematical analysis. In fact, in closed world, we only deal with precisiated information. However, most real world problems cannot satisfy the provable validity condition since they are mostly defined in uncertain, non-stationary, evolving, and imprecise environment and dealt with incomplete information. Therefore, in most cases, the provably-valid solutions cannot be achieved. Zadeh in 2009 introduced extended fuzzy logic (FLe) [2] to deal with the issue of being unprecisiated. As a matter of fact, FLe is a new trend to solve problems by introducing unprecisiated fuzzy logic (FLu) and adding it to fuzzy logic.

At a relatively of high level of abstraction, the philosophy of FLe comes back to this issue that everything in the world is continuously changing; therefore, there are, actually, no right answers. The analysis in FLe to deal with this continuous changing and in the presence of far less precise data is based on quasi-mathematical analysis [3,4]. Within FLe, membership functions and generalized constraints are determined based on perception; in other words, they are not defined as precisiated concepts. The unprecisiated nature in FLe is modeled using f-transformation. f-Transformation is a one-to-many map [2] in which the originality of 'one' is preserved during mapping. This concept plays a major role in lowering the requirement of precision from fuzzy logic still main on right track to solve open world problems that leads to emerge FLe from fuzzy logic. Therefore, in FLe, we face two types of answers: S-answers (relate to specificity) and f-answers (relate to generality) [3]. The S-answer is the centroid of the relevant $\mathrm{f}$ answer that is the f-transformed of the S-answer. It should be noted that since FLe is an imprecise logic of imprecise reasoning, the Sanswer has two degrees that are different from each other originality and structurally and are related to different modules of FLe. The first degree relates to the approach's ability to handle incomplete information while the second degree relates to the validity of the approach that the given problem is handled. Therefore, within FLe, we obtain fuzzy valid results while capturing more uncertainty in reasoning and computation.

Although FLe is very young logic, it has satisfied the engineering inclination as a sound inference theory by showing its applicability in providing promise results for challenging and complex problems such as identification systems [5], decision making [6], and risk assessment [7]. Apart from the mentioned application, the main concern of FLe is its applicability to robotic and artificial intelligence issues. As a matter of fact, building the next generation of robotic systems with the ability of dealing with complex real-world application requires integration between logic, learning, and computation. This can be done within FLe.

In fact, the goal of considering FLe would be to examine how considering validity degree of embedded knowledge structures affects reasoning and modeling for scalable and imprecise data in modeling of risk-sensitive decision-making in complex, highly dynamic, and uncertain environments that a future robot needs to deal.

\section{Reference}

1. Zadeh LA (1965) Fuzzy sets. Information and Control 8: 338-353.

2. Zadeh LA (2009) Toward extended fuzzy logic-A first step. Fuzzy Sets Syst 160: 3175-3181.

3. Sabahi F and Akbarzadeh TMR (2013) A qualified description of extended fuzzy logic. Inform. Sci. 244: 60-74.

4. Sabahi F and Akbarzadeh TMR (2014) A framework for analysis of extended fuzzy logic. Journal of Zhejiang University SCIENCE C 15: 584-591.

5. Sabahi F and Akbarzadeh TMR (2014) Identification of nonlinear systems based on the extended fuzzy logic. Tabriz Journal of Electrical Engineering 44: 23-32

6. Sabahi F and Akbarzadeh TMR (2014) Introducing validity into fuzzy probabilities for judicial decision-making. International Journal of Approximate Reasoning 55: 1383-1403.

7. Sabahi F and Akbarzadeh TMR (2013) Comparative evaluation of risk factors in coronary heart disease based on fuzzy probability-validity modeling. Journal of Zanjan University of Medical Sciences and Health Services 22: 73-83. 